\title{
Baicalin From Scutellaria baicalensis Impairs Th1 Polarization Through Inhibition of Dendritic Cell Maturation
}

\author{
Mi Eun Kim ${ }^{1, \dagger}$, Hyung Keun Kim ${ }^{2, \dagger}$, Hyeon-Young Park ${ }^{1}$, Dae Hyun Kim³ ${ }^{3}$, Hae Young Chung ${ }^{3}$, \\ and Jun Sik Lee ${ }^{1, *}$ \\ ${ }^{1}$ Department of Biology, College of Natural Sciences, Chosun University, Gwangju 501-759, South Korea \\ ${ }^{2}$ Heart Research Center of Chonnam National University Hospital, Cardiovascular Research Institute, \\ Chonnam National University, Gwangju 501-757, Korea \\ ${ }^{3}$ Molecular Inflammation Research Center for Aging Intervention (MRCA), Department of Pharmacy, \\ Pusan National University, Busan 609-735, Korea
}

Received September 2, 2012; Accepted December 17, 2012

\begin{abstract}
Baicalin from Scutellaria baicalensis is a major flavonoid constituent found in the traditional Chinese medicinal herb Baikal skull cap. It has been widely used for the treatment of various diseases such as pneumonia, diarrhea, and hepatitis. Recent studies have demonstrated that baicalin possesses a wide range of pharmacological and biological activities, including anti-inflammatory, anti-microbial, anti-oxidant, and anti-tumor properties. Specifically, its antiinflammatory activity has been estimated in various animal models of acute and chronic inflammation; however, its effects on dendritic cells (DCs) maturation and immuno-stimulatory activities are still unknown. In this study, we attempted to determine whether baicalin could influence DC surface molecule expression, antigen uptake capacity, cytokine production, and capacity to induce T-cell differentiation. Baicalin was shown to significantly suppress the expression of surface molecules CD80, CD86, major histocompatibility complex (MHC) class I, and MHC class II as well as the levels of interleukin-12 production in lipopolysaccharide stimulated DCs. Moreover, baicalin-treated DCs showed an impaired induction of the Thelper type 1 immune response and a normal cell-mediated immune response. These findings provide important understanding of the immunopharmacological functions of baicalin and have ramifications for the development of therapeutic adjuvants for the treatment of DCs-related acute and chronic diseases.
\end{abstract}

Keywords: baicalin, dendritic cell, cytokine, Th1 polarization

\section{Introduction}

An extensive body of research is currently focused on the biological activities of dendritic cells (DCs), specifically regarding their possible clinical use as cellular adjuvants for the treatment of chronic infectious disease, immune homeostasis, and tumors $(1-6)$. DCs are professional antigen presenting cells (APCs) that possess immune sentinel properties and initiate T-cell responses against pathogens and tumors $(7,8)$. The generation of an optimal immune response often requires the presence

\footnotetext{
These authors equally contributed.

*Corresponding author. junsiklee@chosun.ac.kr

Published online in J-STAGE

doi: 10.1254 /jphs.12200FP
}

of $\mathrm{CD}^{+}$and $\mathrm{CD} 8^{+} \mathrm{T}$ cells as well as the expression of T-cell-specific antigens on APCs $(9,10)$. As potent APCs, DCs possess immune sentinel properties, which enable the induction of primary immune responses and the activation of T-cell responses against pathogens and tumors. Immature DCs capture and process exogenous agents within the peripheral tissues where they mature. During maturation, DCs migrate to the lymphoid organs where they stimulate naïve $\mathrm{T}$ cells by signaling through the major histocompatibility complex (MHC) and costimulatory molecules. DCs have also been characterized as highly responsive to both bacterial products and $\mathrm{i}$ nflammatory cytokines such as lipopolysaccharide (LPS) and tumor necrosis factor (TNF)- $\alpha$. DCs located in peripheral tissues generally tend to be both functionally 
and phenotypically immature. Immature DCs do not induce primary immune responses, as they have low expression of co-stimulatory molecules. While in the immature state, DCs have been shown to effectively capture and process exogenous antigens (Ag) within peripheral tissues, where they then begin to mature. Upon stimulation, DC maturation has been associated with high expression of MHC class I and II, decreased or absent Ag uptake, the up-regulation of co-stimulatory molecules, and interleukin (IL)-12 production. Therefore, DC functional modulators could be supported by therapeutic adjuvants for DC-associated immune diseases.

Baicalin is a major flavonoid constituent found in the traditional oriental medicinal herb Baikal skull cap (Scutellaria baicalensis GeorgI) and is known as "HwangKeum" in Korea and "Ogon" in Japan. Baicalin has been widely used for the treatment of various diseases such as hepatitis, pneumonia, allergies, diabetes, and cancer $(11,12)$. Previous studies have demonstrated that baicalin possesses a wide range of biological and pharmacological activities, including anti-inflammatory, antioxidant, and anti-cancer properties $(13-17)$. The mechanism of action indicates that its anti-inflammatory activity is responsible for suppressing the functions of various inflammatory cells such as lymphocytes and inhibiting the production of pro-inflammatory mediators such as IL-6 and TNF- $\alpha(13,17)$. However, until now, the immune cellular targets of baicalin have remained enigmatic, thereby leaving open for discussion future research regarding the issue of the global function of baicalin as related to DCs maturation and immuneregulatory activities.

In the present study, we sought to characterize the effects of a non-cytotoxic dose of baicalin on the functional and maturational properties of murine bone marrow-derived DCs. Our findings demonstrated that baicalin treatment inhibited the phenotypic and functional maturation of DCs. Thus, this available drug may constitute a simple, inexpensive, and highly effective means to exploit the immunostimulatory capacity of DCs.

\section{Materials and Methods}

\section{Animals and chemicals}

Male 8 - 12-week-old C57BL/6 $\left(\mathrm{H}-2 \mathrm{~K}^{\mathrm{b}}\right.$ and I- $\left.\mathrm{A}^{\mathrm{b}}\right)$ and $\mathrm{BALB} / \mathrm{c}\left(\mathrm{H}-2 \mathrm{~K}^{\mathrm{d}}\right.$ and $\left.\mathrm{I}-\mathrm{A}^{\mathrm{d}}\right)$ mice were purchased from the Korean Institute of Chemistry Technology (Daejeon, Korea). They were housed in a specific pathogen-free environment within our animal facility for at least 1 week before use. All mouse work was approved by the IACUC and was performed in our IACUC approved facility. Baicalin was purchased from Sigma (purify was $>99.0 \%$, assessed by HPLC).

\section{Reagents and Abs}

Recombinant mouse (rm) GM-CSF and rmIL-4 were purchased from R\&D Systems (Minneapolis, MN, USA). Dextran-FITC (molecular mass, 40,000) and LPS (from Escherichia coli 055:B5) were obtained from SigmaAldrich (St. Louis, MO, USA). Cytokine ELISA kits for murine IL-12p70, IFN- $\gamma$, IL-4, and IL-2 were purchased from BD Pharmingen (Rockville, MD, USA). FITC- or PE-conjugated $\mathrm{mAbs}$ used to detect the expression of CD11c (HL3), CD80 (16-10A1), CD86 (GL1), IA ${ }^{\mathrm{b}} \beta$ chain (AF-120.1), H2K $\mathrm{K}^{\mathrm{b}}$ (AF6-88.5), or the intracellular expression of IL-12p40/p70 (C15.6), and IL-10 (JESS$16 \mathrm{E} 3$ ) by flow cytometry, as well as isotype-matched control mAbs, were purchased from eBioscience (San Diego, CA, USA).

\section{Generation and culture of DCs}

DCs were generated from murine bone marrow cells. Briefly, bone marrow was flushed from the tibiae and femurs of male C57BL/6 and depleted of red cells with ammonium chloride. The cells were plated in six-well culture plates $\left(10^{6}\right.$ cells $/ \mathrm{ml}, 3 \mathrm{ml} /$ well $)$ in OptiMEM (Invitrogen Life Technologies, Carlsbad, CA, USA) supplemented with $10 \%$ heat-inactivated FBS, 2 mM Lglutamine, $100 \mathrm{U} / \mathrm{ml}$ penicillin, $100 \mu \mathrm{g} / \mathrm{ml}$ streptomycin, $5 \times 10^{-5} \mathrm{M}$ 2-ME, $10 \mathrm{mM}$ HEPES (pH 7.4), $20 \mathrm{ng} / \mathrm{ml}$ rmGM-CSF and rmIL-4 at $37^{\circ} \mathrm{C}, 5 \% \mathrm{CO}_{2}$. On day 3 of the culture, floating cells were gently removed, and fresh medium was added. On day 6 of the culture, nonadherent cells and loosely adherent proliferating DCs aggregates were harvested for analysis or stimulation, or in some experiments, replated in $60-\mathrm{mm}$ dishes $\left(10^{6}\right.$ cells $/ \mathrm{ml} ; 5$ $\mathrm{ml} /$ dish). On day $6,80 \%$ or more of the nonadherent cells expressed CD11c. In certain experiments, to obtain highly purified populations for subsequent analyses, the DCs were labeled with bead-conjugated anti-CD11c $\mathrm{mAb}$ (Miltenyi Biotec, Gladbach, Germany) followed by positive selection through paramagnetic columns (LS columns, Miltenyi Biotec) according to the manufacturer's instructions. The purity of the selected cell fraction was $>95 \%$.

\section{Stimulation of DC by baicalin}

Baicalin dissolved in DMSO or DMSO alone $(0.01 \%$ $\mathrm{v} / \mathrm{v})$ was added to cultures of isolated DCs in six-well plates $\left(10^{6}\right.$ cells $/ \mathrm{ml}, 3 \mathrm{ml} /$ well $)$. DMSO alone was used as a control because it showed no cytotoxicity in DCs. For the analysis of apoptosis, DCs were stimulated with LPS or left unstimulated. Additionally, apoptosis was analyzed over time by staining of phosphatidylserine translocation with FITC-annexin V in combination with a propidium iodine kit according to the manufacturer's instructions. 


\section{Flow cytometric analysis}

On day 6, BM-DC were harvested, washed with phosphate-buffered saline (PBS) and resuspended in fluorescence activated cell sorter (FACS) washing buffer ( $1 \%$ fetal bovine serum in PBS). The cells were first blocked with $10 \%(\mathrm{v} / \mathrm{v})$ normal goat serum for $15 \mathrm{~min}$ at $4^{\circ} \mathrm{C}$ and stained with phycoerythrin (PE)-conjugated anti-H-2K $\mathrm{K}^{\mathrm{b}}$ (MHC class I), anti-I-A ${ }^{\mathrm{b}}$ (MHC class II), anti$\mathrm{CD} 80$, and anti-CD86 with fluorescein isothiocyanate (FITC)-conjugated anti-CD11c (PharMingen, San Diego, CA, USA) for $30 \mathrm{~min}$ at $4^{\circ} \mathrm{C}$. The stained cells were analyzed using a FACSCalibur flow cytometer (BD Biosciences, San Jose, CA, USA).

\section{Cytokines assay}

DCs were first blocked with $10 \%(\mathrm{v} / \mathrm{v})$ normal goat serum for $15 \mathrm{~min}$ at $4^{\circ} \mathrm{C}$ and then stained with FITCconjugated $\mathrm{CD} 11 \mathrm{c}^{+}$antibody for $30 \mathrm{~min}$ at $4^{\circ} \mathrm{C}$. The cells stained with the appropriate isotype-matched Ig were used as negative controls. The cells were fixed and permeabilized with the Cytofix/Cytoperm kit (BD Pharmingen) according to manufacturer's instructions. Intracellular IL-12p40/p70 and IL-10 were stained with fluorescein PE-conjugated antibodies (PharMingen) in a permeation buffer. The cells were analyzed using the FACSCalibur flow cytometer with the CellQuest program. Furthermore, murine IL-12p70 or IL-4 from DC and IL-2, IFN- $\gamma$ as well as IL-4 from co-cultured T cells were measured using an ELISA kit (BD Pharmingen), according to manufacturer's instructions.

\section{Mixed lymphocyte reaction}

Responder T cells were isolated from spleen of BALB/c mice in a MACS column (Miltenyi Biotec). Staining with FITC-conjugated anti-CD4 antibody (BD Pharmingen) revealed that they consisted mainly of $\mathrm{CD}^{+}$cells $(>95 \%)$. DCs were treated with $50 \mu \mathrm{g} / \mathrm{ml}$ mitomycin $\mathrm{C}$ (Sigma) for $1 \mathrm{~h}$ and added in graded doses to $1 \times 10^{5}$ allogeneic T cells in U-bottomed 96well microtiter culture plates. During the last 18 of the 72-h culturing, cell proliferation was quantified by $\left({ }^{3} \mathrm{H}\right)$ thymidine (NEN-DuPont, Boston, MA) uptake of cells by adding $0.5 \mu \mathrm{Ci}$ of (methyl ${ }^{3} \mathrm{H}$ )thymidine in the culture medium. The cells were harvested onto glass fiber filters (Inotech Biosystems, Zurich, Switzerland) and the radioactivity was measured in a scintillation counter. Results are presented as the mean c.p.m. of triplicated cultures.

\section{Statistics}

All results were expressed as the means \pm S.D. of the indicated number of experiments. Statistical significance was estimated using a Student's $t$-test for unpaired obser- vations, and the differences were compared with regard to statistical significance by one-way ANOVA, followed by Bonferroni's post hoc test. The categorical data from the fertility test were subjected to statistical analysis via the Chi-square test. A $P$ of $<0.01$ was considered significant.

\section{Results}

\section{Baicalin inhibits LPS-induced DCs maturation}

In an initial series of experiments, we investigated whether baicalin could influence the maturation of DCs. Bone marrow-derived DCs were cultured for 6 days with supplemented medium including granulocyte-macrophage colony-stimulating factor and IL-4. Bone marrowderived DCs have certain characteristics similar to myeloid DCs and is a major stimulator of T cells instead of plasmacytoid DCs. Different concentrations of baicalin were added to the culture on day 6 with or without 200 $\mathrm{ng} / \mathrm{ml}$ of LPS. Baicalin (Fig. 1A) was determined to be cytotoxic to DCs at concentrations in excess of $30 \mu \mathrm{M}$ (data not shown). There were no marked differences in the percentage of dead cells when the concentrations were all set to $\leq 30 \mu \mathrm{M}$. We then determined the effects of different physiological concentrations of baicalin on DC maturation. To determine whether baicalin has a regulatory effect on DC maturation, we determined the protein expression levels of co-stimulatory molecules B7-1 (CD80), B7-2 (CD86), and MHC class I and II. As shown in Fig. 1B, the treatment with baicalin at various concentrations attenuated the expression of CD80, CD86, MHC class I, and MHC class II on CD11 $\mathrm{c}^{+} \mathrm{DCs}$, which are upregulated during DCs maturation. As shown in Fig. 1C, these molecules were also upregulated within 24 $\mathrm{h}$ of LPS exposure (thick lines). Treatment with $20 \mu \mathrm{M}$ baicalin in the presence of LPS impaired the expression of the MHC class I and II co-stimulatory molecules. Interestingly, a significant downregulation of CD80, CD86, and MHC class I was also observed under these conditions (Fig. 1C, thin lines).

Baicalin impairs the secretion of IL-12 during LPSinduced DCs maturation

It has previously been hypothesized that DCs, macrophages, and monocytes function as sources of proinflammatory molecules such as IL-12 (7). Thus, we examined the ability of baicalin-treated DCs to generate proinflammatory cytokines. IL-12 expression has been identified as a specific marker of DCs activity and the T helper type 1 (Th1) immune response. It has also been considered an important marker for DC maturation and can be used to select Th1 dominant adjuvants. We determined the production of both intracellular IL-12p40/70 
A<smiles>O=C(O)[C@H]1O[C@@H](Oc2cc3oc(-c4ccccc4)cc(=O)c3c(O)c2O)[C@H](O)[C@H](O)[C@@H]1O</smiles>

B
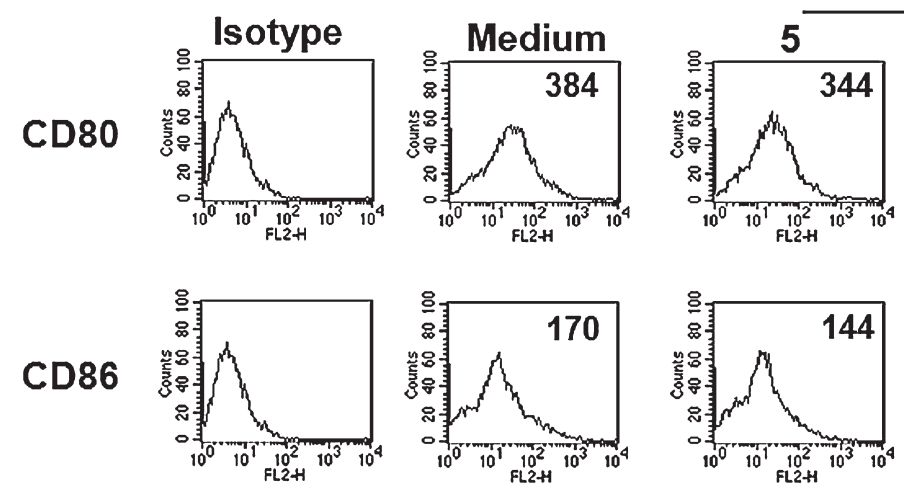

Baicalin $(\mu \mathrm{M})$
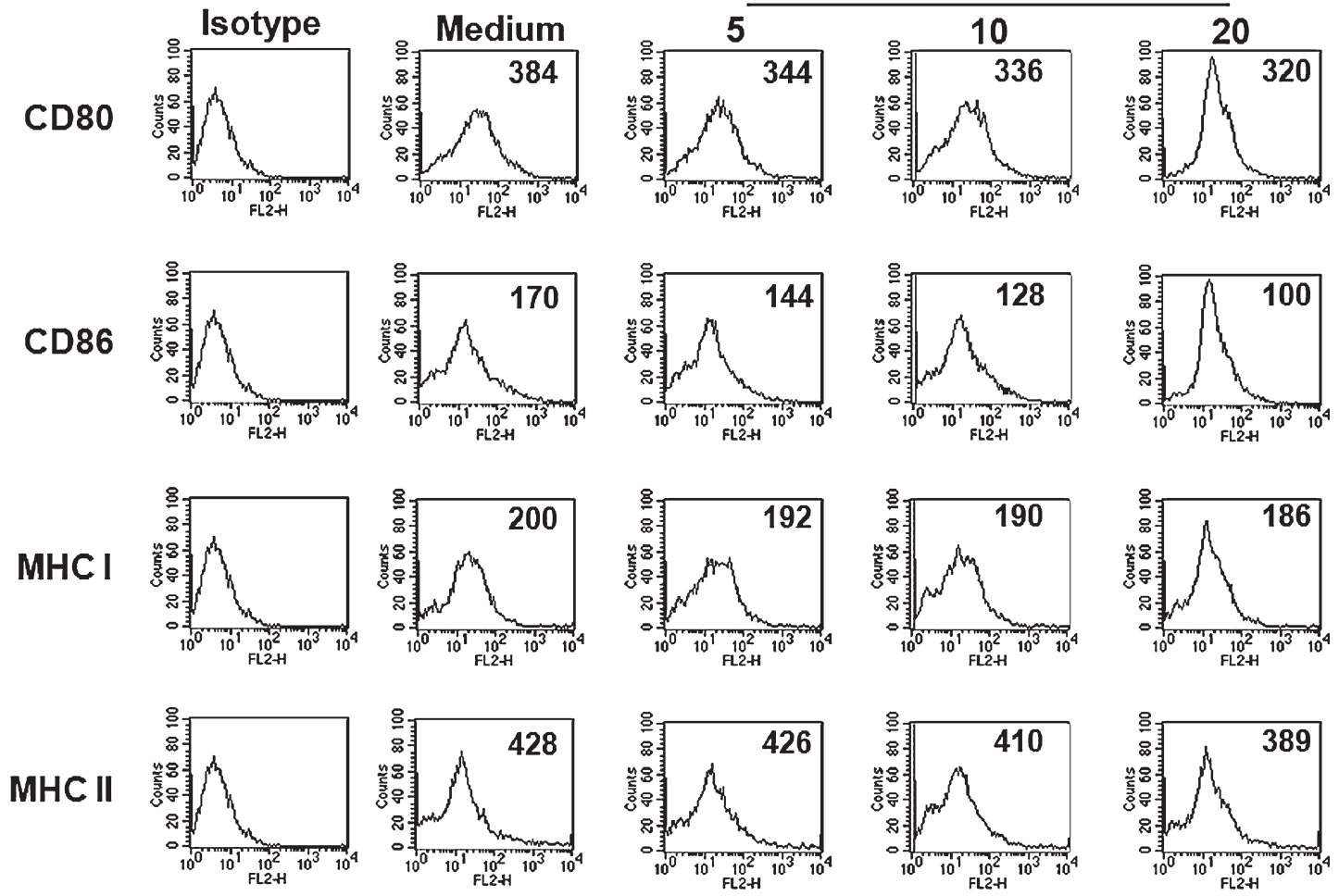

C

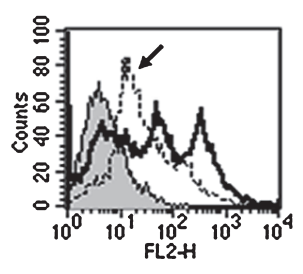

CD80

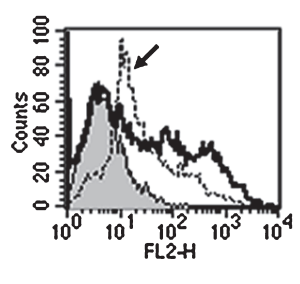

CD86

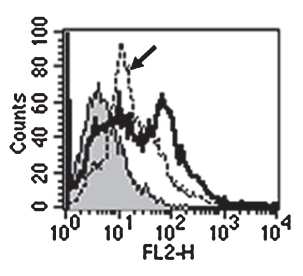

MHC I

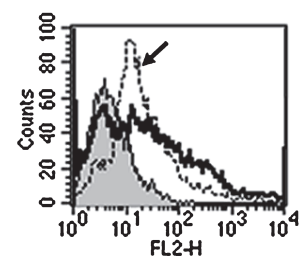

MHC II

Fig. 1. Baicalin inhibits the expression of co-stimulatory molecules MHC class I and II during DCs maturation. The chemical structure of baicalin (A). DCs were generated as described in the Materials and Methods section. On day 6, baicalin was added for $24 \mathrm{~h}$ to the DCs at the concentration of 5, 10, or $20 \mu \mathrm{M}$. The expression of surface molecules was then analyzed by two-color flow cytometry (B). Histograms indicate medium (control) or cells stimulated for $24 \mathrm{~h}$ with $200 \mathrm{ng} / \mathrm{ml}$ LPS in the presence (gray line, isotype control; thick line, LPS treatment; thin line, baicalin plus LPS treatment) of $20 \mu \mathrm{M}$ baicalin on day 6 (C). The results are representative of three identical experiments. 

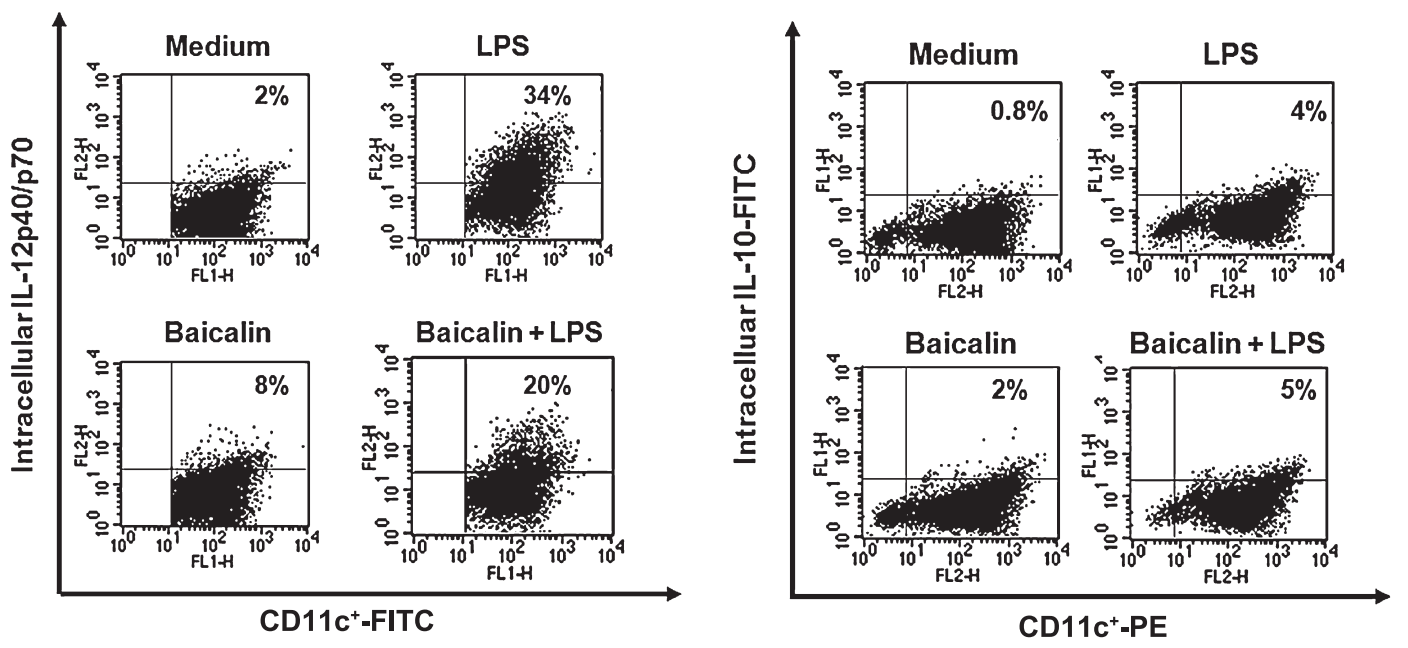

B
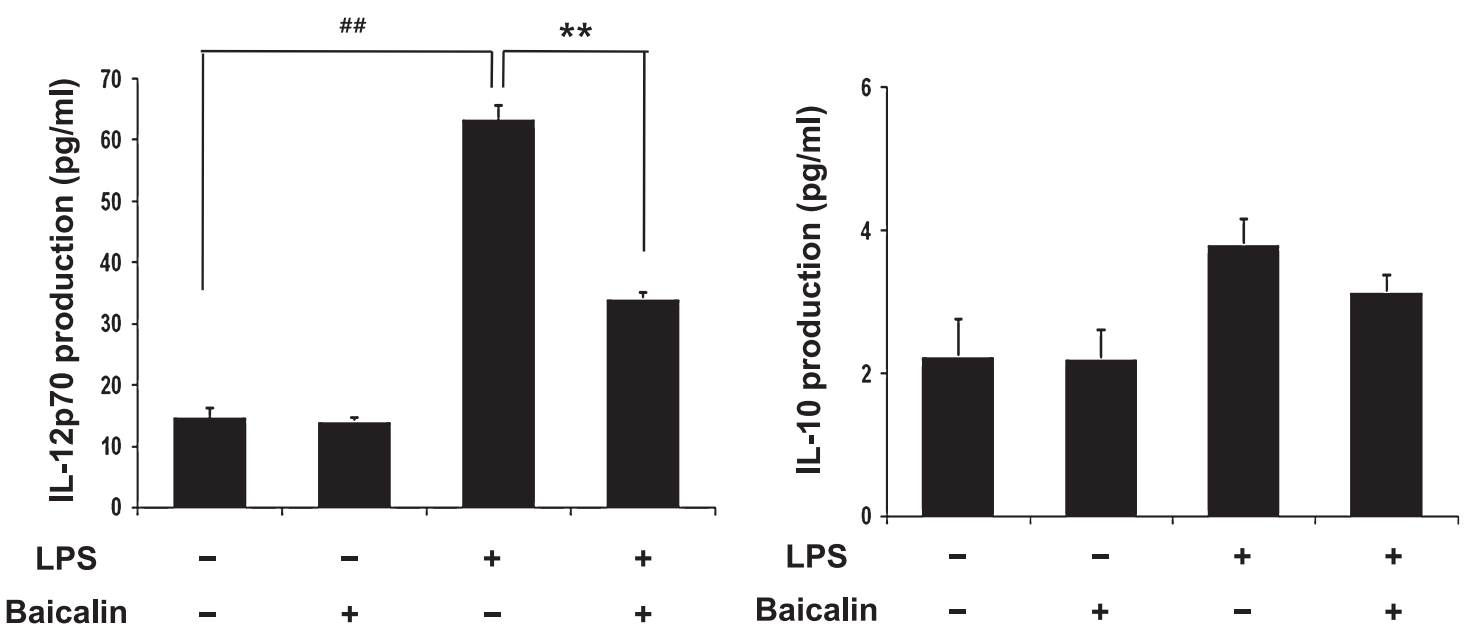

Fig. 2. Baicalin impairs IL-12 production and secretion during LPS-induced DCs maturation. DCs were stimulated by baicalin $(20 \mu \mathrm{M})$ for $24 \mathrm{~h}$ in the absence or presence of LPS. CD11 $\mathrm{c}^{+}$DCs were subsequently analyzed by intracellular cytokine staining (A). The cells were gated for CD11 $\mathrm{c}^{+}$-positive DCs. The DCs $\left(1 \times 10^{5}\right.$ cells $\left./ \mathrm{ml}\right)$ were cultured for $24 \mathrm{~h}$, and the production of bioactive IL-12p70 and IL-10 were analyzed by ELISA from the culture supernatants (B). The results shown are from one representative experiment out of three. ${ }^{\#} P<0.01$ vs. unstimulated DCs (medium only), ${ }^{* *} P<0.01$ vs. LPS-stimulated DCs.

and bioactive IL-12p70 in baicalin-treated DCs. As shown in Fig. 2A, intracellular staining of $\mathrm{CD} 11 \mathrm{c}^{+} \mathrm{DCs}$ with PE-labeled anti-IL-12p40/p70 or FITC-anti-IL-10 mAbs revealed that DCs stimulated with $20 \mu \mathrm{M}$ baicalin expressed IL-12p40/p70 less abundantly than the LPSstimulated DCs, whereas IL-10 was merely detected. When the supernatants were analyzed by enzyme-linked immunosorbent assay (ELISA), IL-10 was also barely detectable $24 \mathrm{~h}$ after stimulation with $200 \mathrm{ng} / \mathrm{ml}$ of LPS. The inhibitory effects of baicalin on the bioactive form of IL-12 (IL-12p70) were also confirmed by ELISA. As shown in Fig. 2B, ELISA analysis revealed high levels of IL-12p70 upon LPS stimulation of DCs $(63.3 \pm 1.4$ $\mathrm{pg} / \mathrm{ml})$ for $24 \mathrm{~h}$, and baicalin $(34 \pm 1.5 \mathrm{pg} / \mathrm{ml})$ attenuated the effects of LPS. These results indicate that baicalin treatment impairs the ability of DCs to produce large amounts of IL-12p70 and suppresses the functional maturation of LPS-stimulated DCs.

\section{Baicalin enhances the immature state of DCs with high} endocytic capacity

In the current results, the expression levels of costimulatory molecules on DCs and the observed changes in IL-12 production indicate that baicalin treatment 
A

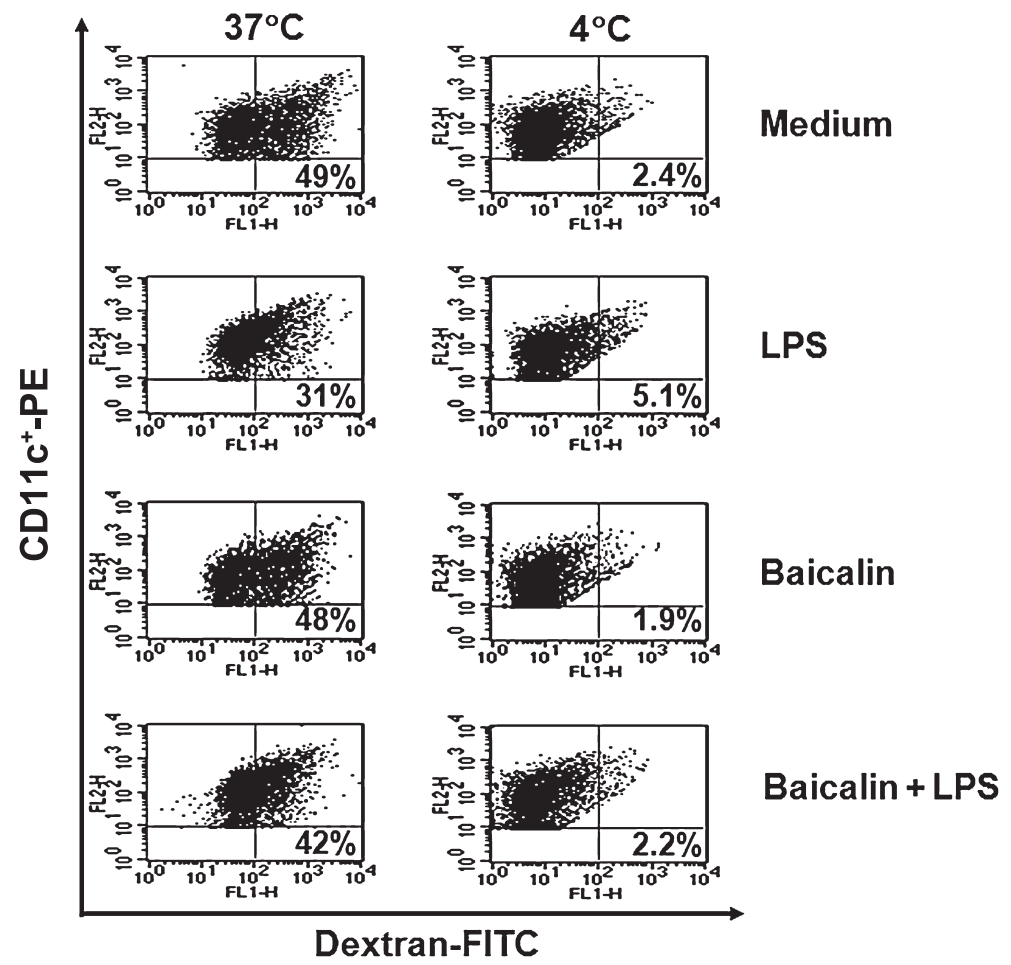

B

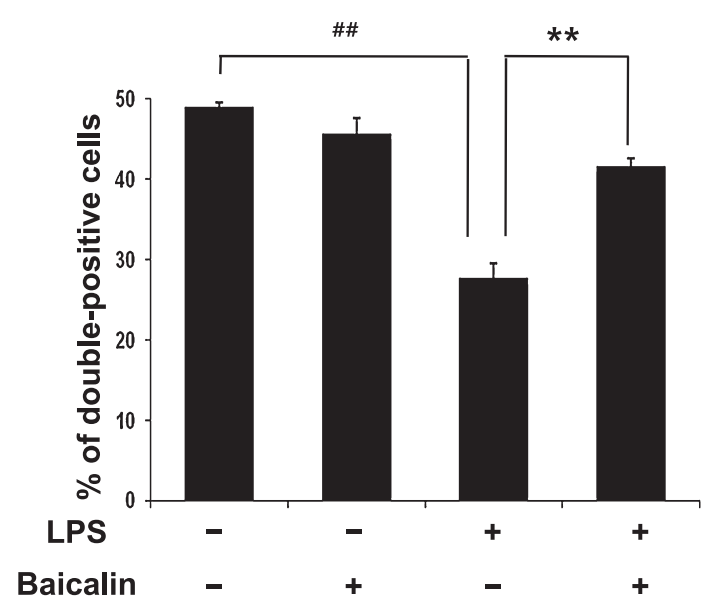

Fig. 3. Baicalin-treated DCs exhibit enhanced endocytic capacity. DCs were treated with $20 \mu \mathrm{M}$ baicalin with or without LPS $(200 \mathrm{ng} / \mathrm{ml})$ for $24 \mathrm{~h}$. The endocytic activity of the DCs was assessed by flow cytometry after FITC-dextran treatment. The DCs were washed and stained with a PE-conjugated anti-CD11 $\mathrm{c}^{+}$antibody. The endocytic activity of the controls was determined after exposure to FITC-dextran at $4^{\circ} \mathrm{C}$. The number represents the percentages of FITC-dextran/CD11 $\mathrm{c}^{+}$-PE double-positive cells (A). The profile of endocytic capacity was also demonstrated as a histogram using the percentages of FITC-dextran/CD11 $\mathrm{c}^{+}-\mathrm{PE}$ doublepositive cells (B). The results shown are from one representative experiment out of three. ${ }^{\#} P<0.01 \mathrm{vs}$. unstimulated DCs (medium only), ${ }^{* *} P<0.01$ vs. LPS-stimulated DCs.

leads to a profound inhibition of DC phenotypic and functional maturation. However, these results did not exclude the possibility that baicalin might induce a general inhibition of the physiological functions of DCs. Thus, we next investigated whether DC stimulation with baicalin altered the ability of the cells to capture AGs. To accomplish this, we treated DC with baicalin in the absence or presence of LPS and added dextran-FITC to the culture media. Based on the uptake of FITCconjugated dextran, the percentage of double-positive cells $\left(\mathrm{CD} 11 \mathrm{c}^{+}-\mathrm{PE}\right.$ with dextran-FITC) did not differ between baicalin-treated and untreated DCs (medium only). Furthermore, the percentage of LPS-stimulated DCs was lower than the percentage of untreated DCs, and the baicalin-treated DCs exhibited a high endocytic capacity for dextran-FITC compared to LPS-stimulated DCs (Fig. 3: A and B). These results indicate that the baicalin-treated DCs were both phenotypically and functionally immature. A set of identical experiments were also performed at $4{ }^{\circ} \mathrm{C}$, and the results of these indicated that the uptake of dextran-FITC by DCs was inhibited at lower temperatures, suggesting that baicalin enhances DC immaturity.

\section{Baicalin impairs DC-mediated induction of Th1 polarization}

To determine whether baicalin inhibits the maturation of DCs into fully functional APCs for APC-mediated allogenic CD4 T-cell stimulation, DCs were treated with baicalin for $24 \mathrm{~h}$ and were subsequently incubated with allogenic CD4 T-cells. As shown in Fig. 4, LPS-treated DCs exhibited more profound proliferation responses than untreated DCs, whereas baicalin appeared to impair the proliferation responses of the allogenic CD4 T-cells elicited by LPS-activated DCs. Therefore, the maturation induced by LPS stimulation profoundly promoted the allostimulatory capacity of the untreated DCs, whereas baicalin treatment significantly impaired the DC allostimulatory capacity. Moreover, given the inhibitory effect of baicalin on DC IL-12 production, a Th1- 

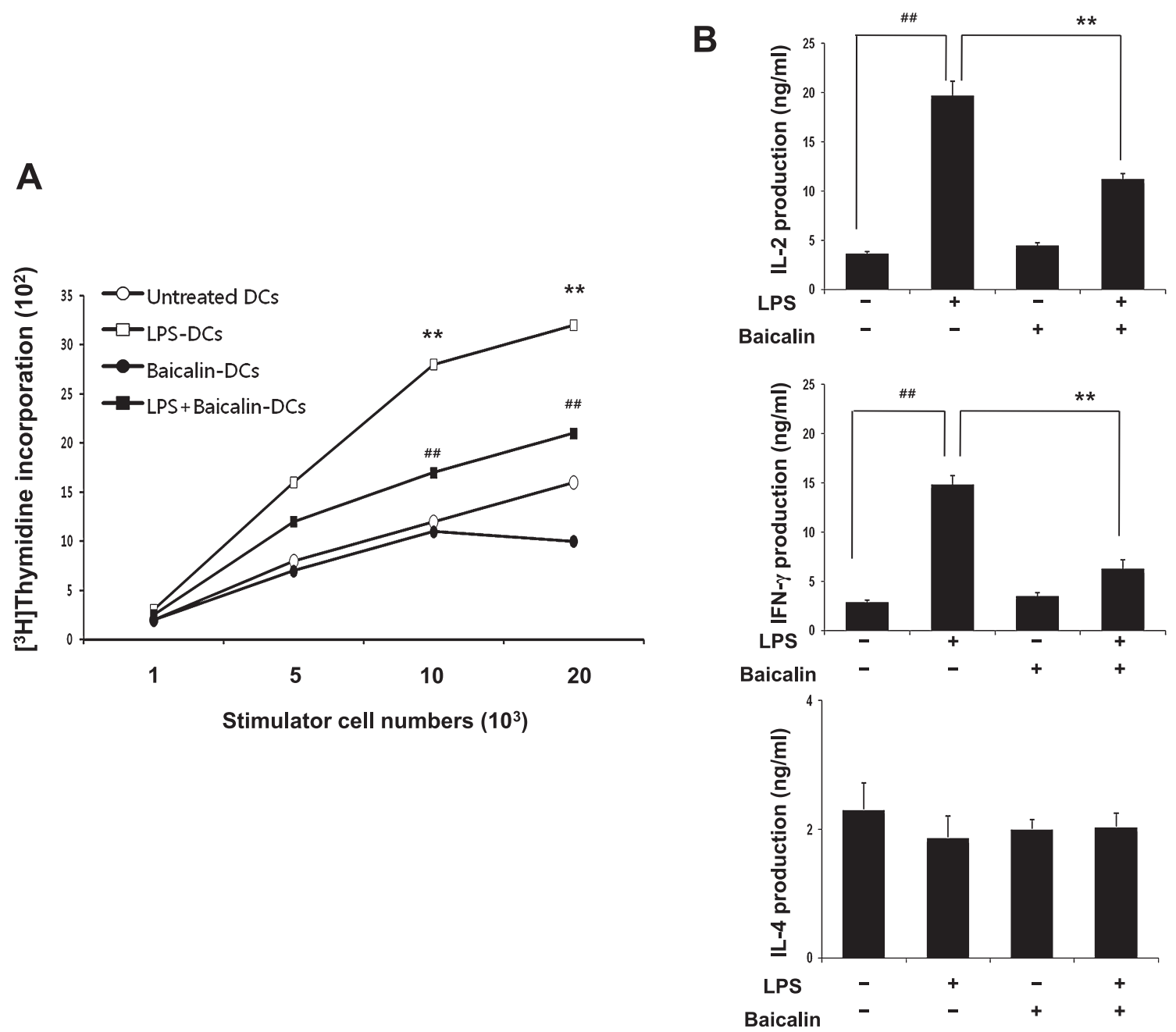

Fig. 4. Baicalin impairs the Th1 response and cytokine production. The DCs were incubated for 24 with medium alone, baicalin $(20 \mu \mathrm{M})$, LPS $(200 \mathrm{ng} / \mathrm{ml})$, or LPS with baicalin. The DCs were washed and then co-cultured with allogenic CD4 T cells. The mixed leukocyte reaction was conducted for 4 days. Background levels of $\left[{ }^{3} \mathrm{H}\right]$ thymidine uptake were determined by measuring reactions without the stimulator (A). The cells were examined for cytokine production via ELISA after $48 \mathrm{~h}$ (B). The results shown are from one representative experiment out of three. ${ }^{\#} P<0.01$ vs. unstimulated DCs (medium only), $* * P<0.01$ vs. LPSstimulated DCs.

inducing cytokine, we next attempted to characterize the primary T-cell response induced by DCs that had matured in the presence of baicalin. Naïve allogenic CD4 T-cells primed by mature DCs differentiated into Th1 lymphocytes, which generated high levels of IFN- $\gamma$ and low levels of IL-4 production. In contrast, CD4 T cells primed with DCs that had matured in the presence of baicalin displayed inhibited IFN- $\gamma$ and IL-2 production (Fig. 4B). These results indicated that the majority of the effects of baicalin on the T-cell differentiating abilities of DCs were the consequence of the inhibition of IL-12 production.

\section{Discussion}

In this study, we investigated the effects of baicalin on the phenotypic and functional maturation of bone marrow-derived DCs. DCs are known to play important roles in immunotherapy and the establishment of hypersensitivity and transplantation tolerance $(18-21)$. Baicalin is a major flavonoid constituent, and many researchers have shown that baicalin exerts a variety of biological effects, including anti-inflammatory and anti-cancer properties $(13,16,17,22)$. Nevertheless, few reports showing the immunoregulatory effect of baicalin have been published. Zhang et al. has reported that Baicalin induced DCs apoptosis in vitro at $50 \mu \mathrm{M}$ treat- 
ment (23). However, we found no marked differences in the percentage of dead cells when the concentrations were all set to $\leq 30 \mu \mathrm{M} / \mathrm{ml}$. It was might due to different sources of baicalin from different companies and cell culture systems. Some research has shown that baicalin promotes regulatory T-cell differentiation through tolllike receptor 4 to modulate $\mathrm{T}$ and $\mathrm{B}$ cells $(24,25)$. However, the exact baicalin modulation pathways that mediate changes in DCs, which are potential APCs and interact with $\mathrm{T}$ cells for cell-mediated immune responses, are still unclear. Moreover, the effects of baicalin on the maturation and immunological response of DCs remain largely unknown. Because of this, we investigated baicalin-exposed DCs with regard to their capacity to sensitize recipients for cell-mediated immune responses. We used a variety of functional assays to ascertain the function and phenotype of baicalin-treated DCs and also determined the DC-mediated immune response in terms of Th1 or Th 2 polarization. To be sure the observed effects of baicalin could be attributed to DCs and not to contaminating cells in the bone marrow-derived cell cultures, the DCs were purified at greater than $95 \%$. Our results indicated that baicalin is a potent inhibitor of LPS-induced DC maturation and impairs the Th1 immune response and provide new insights into the immunopharmacological potential of baicalin.

During maturation, DCs upregulate co-stimulatory molecules, such as CD80, CD86, and MHC class I and II, allowing for effective AG presentation to naïve T cells. Moreover, mature DCs produce and secrete specific cytokines to activate innate effector cells and direct the development and differentiation of specific $\mathrm{T}$ helper cell subsets. As shown in Fig. 1, baicalin inhibited LPS-induced DC maturation. Zhang et al. reported that baicalin had no effect on the expression levels of CD80 and CD86 on LPS (500 ng/ml)-stimulated DCs (23). However, we found that treatment with $20 \mu \mathrm{M}$ baicalin in the presence of LPS $(200 \mathrm{ng} / \mathrm{ml})$ impaired the expression of the CD80 and CD86 as well as MHC class I and II molecules.

The suppressive effects of baicalin on DC maturation can likely be attributed to a non-specific inhibitory effect. Therefore, we assessed the ability of baicalintreated DCs to uptake FITC-dextran via mannose receptor-mediated endocytosis (Fig. 3). As a result, the endocytic capacity of the baicalin-treated DCs was profoundly elevated, suggesting that baicalin suppressed both the phenotypic and functional maturation of DCs.

The helper T-cell-mediated immune response toward Th1 or Th2 polarization depends on the specific cytokines secreted by the activated DCs. High levels of IL-12 will induce differentiation of naïve $\mathrm{CD} 4^{+} \mathrm{T}$ cells into $\mathrm{Th} 1$ cells and simultaneously block the development of the
Th2 lineage (26). The secretion of bioactive IL-12p70 requires the coordinated expression of two of its subunits, namely p35 and p40, which are encoded by two separate genes and are regulated independently. As shown in Fig. 2, DCs enhanced IL-12 production and secreted the bioactive form IL-12p70 in the presence of LPS, and baicalin exerted inhibitory effects on the production of intracellular IL-12p40/p70 and bioactive IL-12p70. In contrast, baicalin and LPS had no effect on IL-10 production in DCs.

Our study also demonstrated that LPS-stimulated DCs skewed naïve $\mathrm{CD} 4^{+} \mathrm{T}$ cells toward developing into IFN$\gamma$-producing $\mathrm{T}$ cells for Th1 polarization. Naïve $\mathrm{CD} 4^{+}$ $\mathrm{T}$ cells stimulated by baicalin-treated DCs generated lower IFN- $\gamma$ levels, but did not alter IL-4 production. Therefore, baicalin significantly impaired the capacity of these cells to proliferate and initiate the Th1 response (Fig. 4). This suggests that baicalin is a potent immunemodulator of the Th1 response, and the inhibition of DCmediated Th1 polarization might constitute a baicalinassociated immunosuppressive mechanism. Consequently, baicalin-mediated inhibition of IL-12 generation in LPS-stimulated DCs may also contribute to the induction of an immunosuppressive state.

In conclusion, we have characterized a variety of baicalin-mediated effects in DCs. Baicalin inhibited the phenotypic cytokine production in DCs, resulting in the inhibition of T-cell activation. Our current data also show that baicalin affected the capability of DCs to determine Th1/Th2 polarization through the regulation of IL-12 and IFN- $\gamma$ production in DCs. These findings underline the immunopharmacological functions of baicalin and suggest a highly effective means for modulating the immunoregulatory capacity of DCs. Taken together, our results suggest new implications for manipulating DC function for potential immunotherapeutic applications.

\section{Acknowledgments}

We thank the Aging Tissue Bank for providing research materials for the study. This work was supported by the Basic Science Research Program through the National Research Foundation of Korea (NRF) funded by the Ministry of Education, Science, and Technology (MEST) (2011-0014137).

\section{References}

1 van de Laar L, Coffer PJ, Woltman AM. Regulation of dendritic cell development by GM-CSF: molecular control and implications for immune homeostasis and therapy. Blood. 2012;119: 3383-3393.

2 Joshi N, Duhan V, Lingwal N, Bhaskar S, Upadhyay P. Adjuvant properties of thermal component of hyperthermia enhanced transdermal immunization: effect on dendritic cells. PLoS One. 
2012;7:e32067.

3 Mattsson J, Yrlid U, Stensson A, Schon K, Karlsson MC, Ravetch JV, et al. Complement activation and complement receptors on follicular dendritic cells are critical for the function of a targeted adjuvant. J Immunol. 2011;187:3641-3652.

4 Zeng J, Xia S, Zhong W, Li J, Lin L. In vitro and in vivo effects of ketamine on generation and function of dendritic cells. J Pharmacol Sci. 2011;117:170-179.

5 Machida Y, Kitamoto K, Izumi Y, Shiota M, Uchida J, Kira Y, et al. Renal fibrosis in murine obstructive nephropathy is attenuated by depletion of monocyte lineage, not dendritic cells. J Pharmacol Sci. 2010;114:464-473.

6 Liu H, Wang S, Sun A, Huang D, Wang W, Zhang C, et al. Danhong inhibits oxidized low-density lipoprotein-induced immune maturation of dentritic cells via a peroxisome proliferator activated receptor gamma-mediated pathway. J Pharmacol Sci. 2012;119:1-9.

7 Lee JS, Jung ID, Jeong YI, Lee CM, Shin YK, Lee SY, et al. D-pinitol inhibits Th1 polarization via the suppression of dendritic cells. Int Immunopharmacol. 2007;7:791-804.

8 Markey KA, Banovic T, Kuns RD, Olver SD, Don AL, Raffelt $\mathrm{NC}$, et al. Conventional dendritic cells are the critical donor APC presenting alloantigen after experimental bone marrow transplantation. Blood. 2009;113:5644-5649.

9 Li L, Kim S, Herndon JM, Goedegebuure P, Belt BA, Satpathy AT, et al. Cross-dressed CD8alpha+/CD103+ dendritic cells prime CD8+ T cells following vaccination. Proc Natl Acad Sci U S A. 2012;109:12716-12721.

10 Vinuesa CG, Linterman MA, Goodnow CC, Randall KL. T cells and follicular dendritic cells in germinal center B-cell formation and selection. Immunol Rev. 2010;237:72-89.

11 Jung HS, Kim MH, Gwak NG, Im YS, Lee KY, Sohn Y, et al. Antiallergic effects of Scutellaria baicalensis on inflammation in vivo and in vitro. J Ethnopharmacol. 2012;141:345-349.

12 Waisundara VY, Hsu A, Huang D, Tan BK. Scutellaria baicalensis enhances the anti-diabetic activity of metformin in streptozotocininduced diabetic Wistar rats. Am J Chin Med. 2008;36:517-540.

13 Lin L, Wu XD, Davey AK, Wang J. The anti-inflammatory effect of baicalin on hypoxia/reoxygenation and TNF-alpha induced injury in cultural rat cardiomyocytes. Phytother Res. 2010;24:429 437.

14 Shen YC, Chiou WF, Chou YC, Chen CF. Mechanisms in mediating the anti-inflammatory effects of baicalin and baicalein in human leukocytes. Eur J Pharmacol. 2003;465:171-181.
15 Zhou QM, Wang S, Zhang H, Lu YY, Wang XF, Motoo Y, et al. The combination of baicalin and baicalein enhances apoptosis via the ERK/p38 MAPK pathway in human breast cancer cells. Acta Pharmacol Sin. 2009;30:1648-1658.

16 Franek KJ, Zhou Z, Zhang WD, Chen WY. In vitro studies of baicalin alone or in combination with Salvia miltiorrhiza extract as a potential anti-cancer agent. Int J Oncol. 2005;26:217-224.

17 Zhu J, Wang J, Sheng Y, Zou Y, Bo L, Wang F, et al. Baicalin improves survival in a murine model of polymicrobial sepsis via suppressing inflammatory response and lymphocyte apoptosis. PLoS One. 2012; 7:e35523.

18 Aspord C, Leccia MT, Salameire D, Laurin D, Chaperot L, Charles J, et al. HLA-A(*)0201(+) plasmacytoid dendritic cells provide a cell-based immunotherapy for melanoma patients. J Invest Dermatol. 2012;132:2395-2406.

19 Palucka K, Banchereau J. Cancer immunotherapy via dendritic cells. Nat Rev Cancer. 2012;12:265-277.

20 Bianco NR, Kim SH, Ruffner MA, Robbins PD. Therapeutic effect of exosomes from indoleamine 2,3-dioxygenase-positive dendritic cells in collagen-induced arthritis and delayed-type hypersensitivity disease models. Arthritis Rheum. 2009;60:380 389.

21 Land WG. Emerging role of innate immunity in organ transplantation part II: potential of damage-associated molecular patterns to generate immunostimulatory dendritic cells. Transplant Rev (Orlando). 2012;26:73-87.

22 Ikezoe T, Chen SS, Heber D, Taguchi H, Koeffler HP. Baicalin is a major component of PC-SPES which inhibits the proliferation of human cancer cells via apoptosis and cell cycle arrest. Prostate. 2001;49:285-292.

23 Zhang H, Jiao Q, Gong Q, Zhang Y, Zhang W, Hu Z. Baicalin induced dendritic cell apoptosis in vitro. Front Pharmacol. 2011;2:15.

24 Gong SQ, Sun W, Wang M, Fu YY. Role of TLR4 and TCR or BCR against baicalin-induced responses in $\mathrm{T}$ and $\mathrm{B}$ cells. Int Immunopharmacol. 2011;11:2176-2180.

25 Yang J, Yang X, Li M. Baicalin, a natural compound, promotes regulatory T cell differentiation. BMC Complement Altern Med. 2012;12:64.

26 Sanecka A, Ansems M, Prosser AC, Danielski K, Warner K, den Brok MH, et al. DC-STAMP knock-down deregulates cytokine production and T-cell stimulatory capacity of LPS-matured dendritic cells. BMC Immunol. 2011;12:57. 